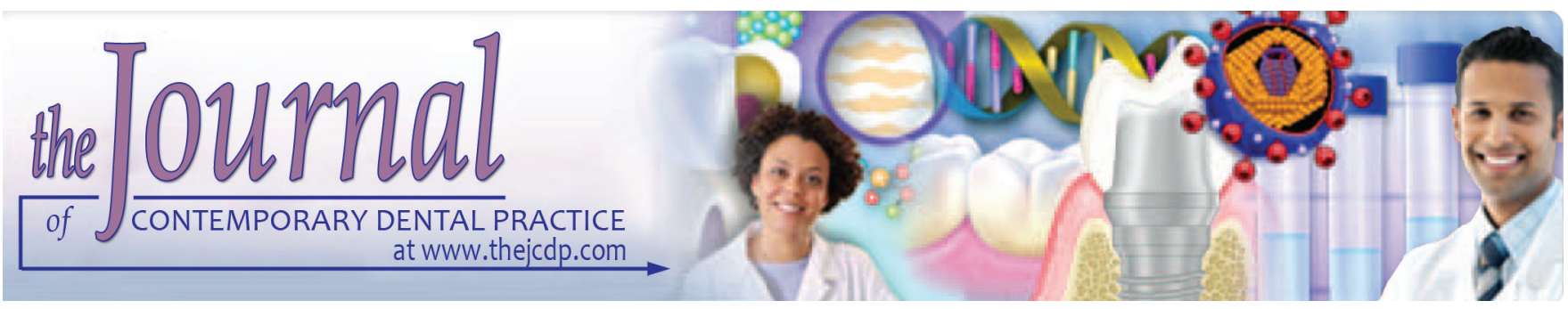

\title{
Cone Beam Computed Tomography-based Evaluation of the Anterior Teeth Position Changes obtained by Passive Self-ligating Brackets
}

\author{
${ }^{1}$ Fernando K Rhoden, ${ }^{2}$ Liliana Á Maltagliati, ${ }^{3}$ Ana C de Castro Ferreira Conti, ${ }^{4}$ Renata R Almeida-Pedrin \\ ${ }^{5}$ Leopoldino C Filho, ${ }^{6}$ Maurício de Almeida Cardoso
}

\begin{abstract}
Aim: The objective of this study was to evaluate the anterior teeth position changes obtained by passive self-ligating brackets using cone beam computed tomography (CBCT).

Materials and methods: Twenty patients with a mean age of 16.5 years, class I malocclusion, constricted maxillary arch, and teeth crowding above $5 \mathrm{~mm}$ were enrolled in this study, and treated by passive orthodontic self-ligating brackets. A sequence of stainless steel thermoset wire was implemented with ending wire of $0.019^{\prime \prime} \times 0.025 "$. The CBCT and dental casts were obtained prior to the installation of orthodontic appliances (T1), and 30 days after rectangular steel wire 0.019 " $\times 0.025$ " installation (T2). The measurements in CBCT were performed with the Anatomage software, and the dental casts were evaluated with a digital caliper rule with an accuracy of $0.01 \mathrm{~mm}$.
\end{abstract}

Results: The CBCT data demonstrated mean buccal inclination of the upper and lower central incisors ranging from $6.55^{\circ}$ to $7.24^{\circ}$ respectively. The upper and lower lateral incisors ranged from $4.90^{\circ}$ to $8.72^{\circ}$ respectively. The lower canines showed an average increase of $3.88^{\circ}$ in the buccal inclination and $1.96 \mathrm{~mm}$ in the transverse intercuspal distance. The upper canines showed a negative inclination with mean average of $-0.36^{\circ}$, and an average increase of $0.82 \mathrm{~mm}$ in the transverse distance, with negative correlation with the initial crowding.

Conclusion: Treatment with passive self-ligating brackets without obtaining spaces increases buccal inclination of the upper and lower incisors with no correlation with the amount of initial teeth crowding. The intercanine distance tends to a small increase showing different inclinations between the arches.

\footnotetext{
1,3-6 Department of Orthodontics, Sagrado Coração University Bauru, São Paulo, Brazil

${ }^{2}$ Private Practice, São Paulo, Brazil

Corresponding Author: Ana C de Castro Ferreira Conti Assistant Professor, Department of Orthodontics, Sagrado Coração University, Bauru, São Paulo, Brazil, Phone: +551432235217, e-mail: accfconti@uol.com.br
}

Clinical significance: When taking into account the selfligating brackets, the amount of initial dental crowding is not a limitation factor that could increase the buccal inclination of the anterior teeth.

Keywords: Cone beam computed tomography, Malocclusion, Orthodontic brackets, Tooth movement.

How to cite this article: Rhoden FK, Maltagliati LÁ, de Castro Ferreira Conti AC, Almeida-Pedrin RR, Filho LC, de Almeida Cardoso M. Cone Beam Computed Tomography-based Evaluation of the Anterior Teeth Position Changes obtained by Passive Selfligating Brackets. J Contemp Dent Pract 2016;17(8):623-629.

Source of support: Nil

Conflict of interest: None

\section{INTRODUCTION}

Orthodontic treatment performed in the permanent dentition in patients with class I malocclusion, and constricted maxillary arch associated with moderate crowding, requires an increase in arch perimeter without teeth extraction. ${ }^{1-4}$ This is achieved in both arches by means of incisors proclination and transverse expansion using conventional or self-ligating brackets to allow achievement of arch alignment and leveling. ${ }^{2,5,6}$ Self-ligating brackets can be separated into two categories: (1) Active and (2) passive, according to their closure mechanism. Active self-ligating brackets have a spring clip storing energy to press against the archwire. Passive self-ligating brackets have a slide that can be closed which does not encroach on the slot lumen, exerting no active force on the archwire. ${ }^{7,8}$ Self-ligating brackets have some advantages over the conventional brackets including: (1) Low static frictional resistance, (2) improved oral hygiene, (3) reduced chair and therapeutic time, (4) full and stable archwire engagement, (5) prolonged therapeutic intervals, and (6) preserved anchorage. ${ }^{9-12}$ It is well known 
that both self-ligating and conventional ligating brackets when used for nonextraction treatment of dental crowding result in dentoalveolar expansion. The amount of transversal increase depends on the mechanics applied depending on the case. ${ }^{4,13-16}$

After the introduction of Damon self-ligating brackets, ${ }^{17}$ it was stated that a passive expansion of the dental arches would be possible by means of tooth movement in a lateral direction with slight forward movement. Furthermore, the thermoset wire with light forces would not be able to withstand the action of the orbicularis muscle of the mouth. As passive self-ligating brackets have free wire movement inside the bracket slot, the wires would slip in a posterior direction according to the crowding resolution, reducing the forward movement. ${ }^{18}$ On the contrary, Vajaria et $\mathrm{al}^{4}$ showed similar posttreatment results in the lower incisors in patients treated with Damon brackets or conventional brackets. Accordingly, Cattaneo et $\mathrm{al}^{2}$ stated that the increase in the arch perimeter is generated by means of buccal inclination of the incisors, and these increments in the arch perimeter are higher when treated with passive self-ligated brackets.

The use of lateral teleradiography has some disadvantages in the measurement of dental inclinations, due to the relative inaccuracy in the cephalometric points tracing required for the delimitation of the incisors and canines long axis, as well as the radiographic images superposition, in which teeth inclination evaluation are difficult to achieve. ${ }^{19}$ With regard to the panoramic radiography, image distortion and magnification are common characteristics, and besides cephalometric points are imprecisely measured with this technique. ${ }^{20}$ For these reasons, quantification of treatment outcome in twodimensional (2D) images raised some criticisms because its reliability was questioned..$^{21}$ Indeed, to fully access the dento-skeletal changes occurring during orthodontic treatment, cone beam computed tomography (CBCT) datasets are required. The $\mathrm{CBCT}$ dataset allows measurements on individual CT slices, making three-dimensional (3D) assessments of dento-skeletal changes much more precisely with a significant lower effective radiation dose compared to $2 \mathrm{D}$ techniques. ${ }^{2,20,22}$

Here, we aimed to evaluate by means of CBCT changes in anterior teeth inclination from orthodontic treatment with passive self-ligating brackets. Moreover, we aimed to determine how much crowding could influence the inclination movement and intercanine distance, which was measured in dental casts.

\section{MATERIALS AND METHODS}

This randomized, prospective, and controlled clinical study was approved by the Ethics Committee on
Human Research of the Sagrado Coração University, under protocol number 236/11. Twenty patients from the Orthodontic Department were enrolled in this study. The inclusion criteria were as follows: Between 13 and 19 years of age (mean age of 16.5 years) at the start of the treatment, with complete dentition, class I malocclusion with moderate dental crowding in the upper and lower arches (between 5.0 and $7.0 \mathrm{~mm}$ ), calculated by the irregularity index. ${ }^{23}$ Moreover, patients were excluded if they had previous orthodontic treatment, missing teeth in the anterior region, cleft lip and palate, or other severe skeletal discrepancy and/or syndromes. Written informed consent to undergo the CBCT radiographic examination was obtained prior to initial treatment from all patients. Dental casts and CBCT scans (i-Cat Imaging Sciences International, Hatfield, Pennsylvania, USA) were obtained prior to the initial orthodontic treatment (T1) and after treatment completion (T2) from all patients. The CBCT scans were adjusted as follows: $120 \mathrm{KvP}, 36 \mathrm{~mA}$, 20 seconds of exposure time, $8 \mathrm{~cm}$ of field of view (FOV) with a $0.2 \mathrm{~mm}$ isotropic voxel size. One blinded examiner performed all the measurements.

The same sequence of treatment has been followed for all patients, without any procedure to achieve additional space or any approach to modify the alignment and leveling. The passive self-ligating brackets Smart Clip (3M, Monrovia, USA) with McLaughlin, Bennett and Trevisi (MBT) prescription were bonded using as reference the $\mathrm{EV}$ point in the center of the clinical crown, and the first and second molars received bonding tubes. The treatment protocol adopted the following sequence of nickel-titanium thermo-activated wire: $0.014^{\prime \prime} \times 0.016^{\prime \prime}$, $0.016^{\prime \prime} \times 0.022^{\prime \prime}, 0.019^{\prime \prime} \times 0.025^{\prime \prime}$. The alignment and leveling has been finished with $0.019^{\prime \prime} \times 0.025^{\prime \prime}$ stainless steel wire. Each wire was maintained for at least 10 weeks or until the next wire could be introduced without difficulty. The $0.019^{\prime \prime} \times 0.025^{\prime \prime}$ stainless steel wire was also maintained for at least 10 weeks to allow the release torque. At this stage, final dental casts and CBCT scans were achieved (T2).

The volumetric image data were converted to Digital Imaging and Communications in Medicine (DICOM) format and imported in the in vivo Dental Application software (Anatomage, Version 5.3.1, San Jose, CA) to generate $3 \mathrm{D}$ and multiplanar reconstructed images. The measurements were made in $2.5 \mathrm{~mm}$ thick sagittal slices, obtained according to the following protocol: (1) Initially, the patient's head was adjusted to determine the palatal and mandibular planes; (2) in the sagittal and coronal sections, the inclination measurements were taken from the upper and lower incisors and canines respectively (Figs 1A and B). The orientation for evaluating the teeth inclination was the tooth long axis, having the apex and the incisal cusp as reference point in relation to the 

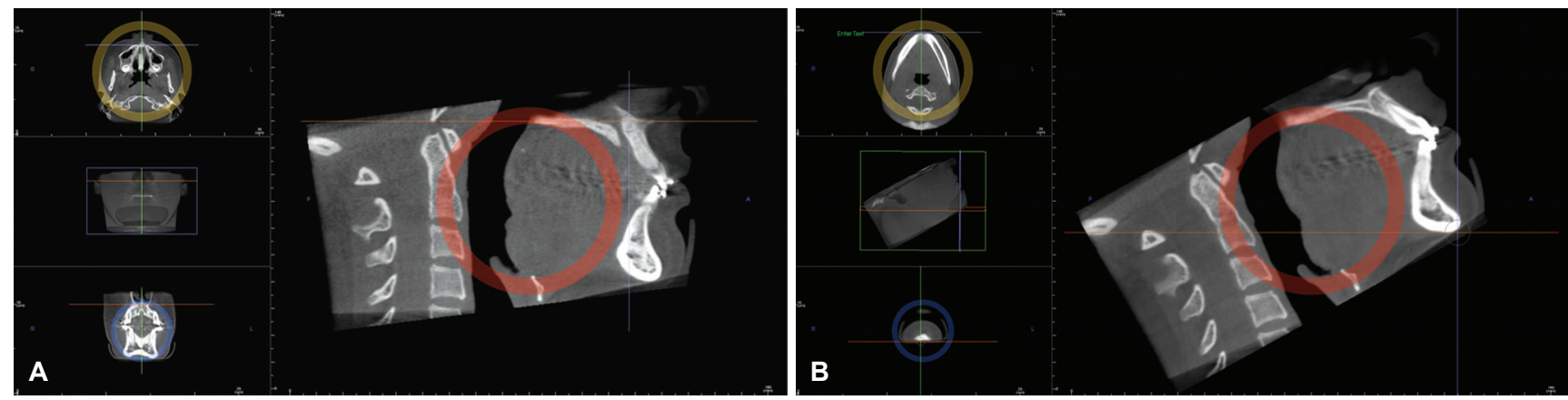

Figs $1 \mathrm{~A}$ and B: For all measurements, volumetric image data were converted to DICOM format and imported in the Anatomage software to generate 3D and multiplanar reconstructed images: (A and B) To establish the maxillary and mandibular plane respectively. The head was manipulated in three directions, so that the horizontal reference line stay superimposed on the palatal plane in sagittal position

respective maxillary and mandibular planes (Figs 2A to D). Importantly, the $2.5 \mathrm{~mm}$ thick axial and sagittal slices were obtained to prevent manipulation in the axial and sagittal directions, which in turn would alter the patient's position in relation to palatal and/or mandibular plans stipulated.

The dental cast's measurements were performed on 40 pairs of casts $[20$ pairs at the beginning (T1) and
20 pairs obtained at least 10 weeks after $0.019^{\prime \prime} \times 0.025^{\prime \prime}$ wire steel installation (T2)] with a calibrated digital caliper rule (Mitutoyo pachymeter, Japan) with $0.01 \mathrm{~mm}$ of accuracy. The caliper was placed parallel to the occlusal plane (Figs $3 \mathrm{~A}$ to $\mathrm{C}$ ), and the analysis of changes in dental alignment was based on the irregularity index of $\operatorname{Little}^{23}$ and intercanine distance, ${ }^{24}$ as described.
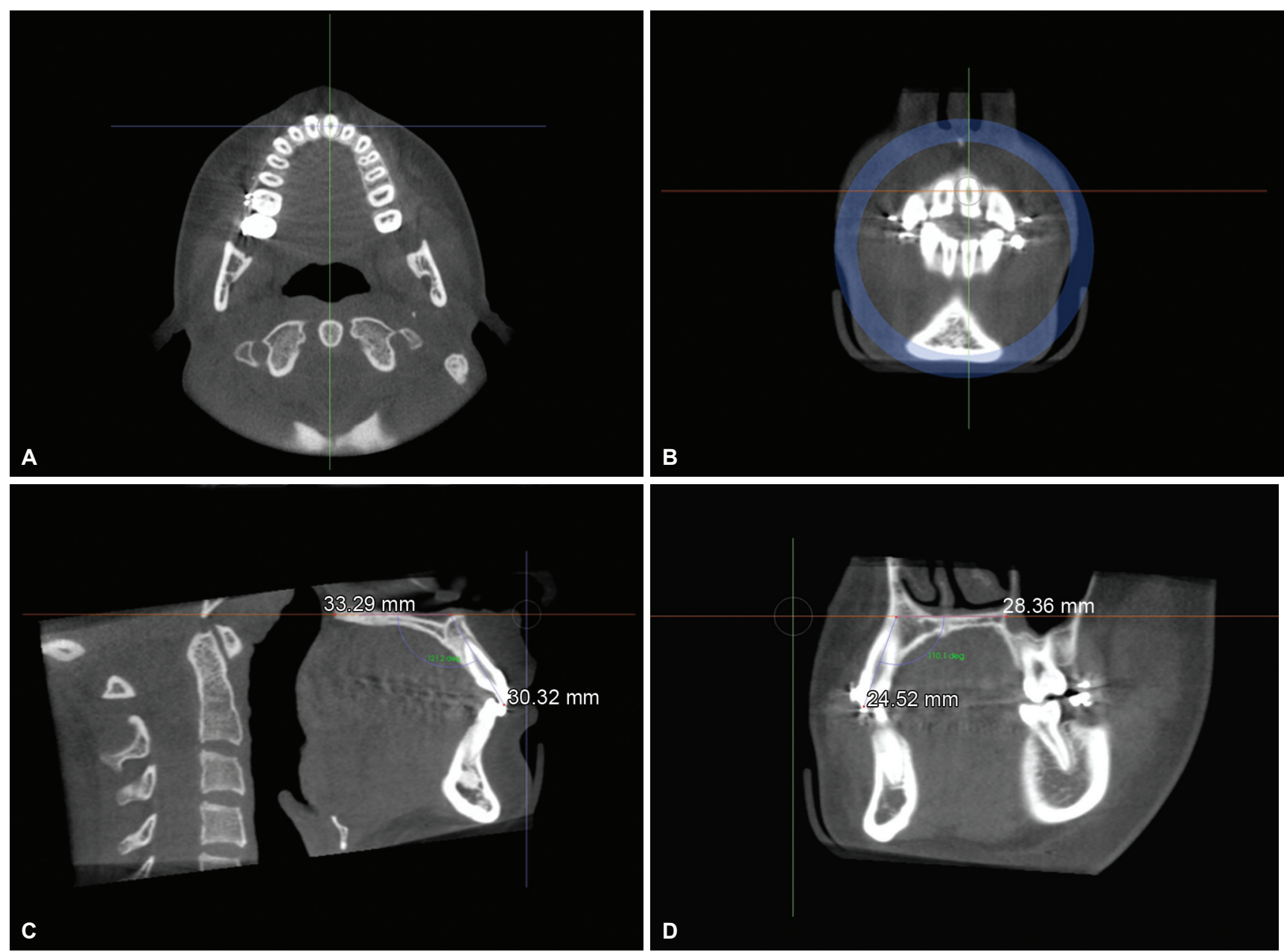

Figs 2A to D: Representative 3D reconstructed images in axial (A), Coronal (B), sagittal (C) slices. In sagittal sections (C), the inclination measurements of the upper and lower incisors were taken. In coronal sections (D) the inclination measurements of upper and lower canines were measured. The reference for evaluating the dental inclination was the tooth long axis, having reference points the cusp apex or incisal edge in the respective maxillary and mandibular planes. Sagittal (C) and coronal (D) sections showing representative images for evaluation of incisors and canine inclinations respectively 


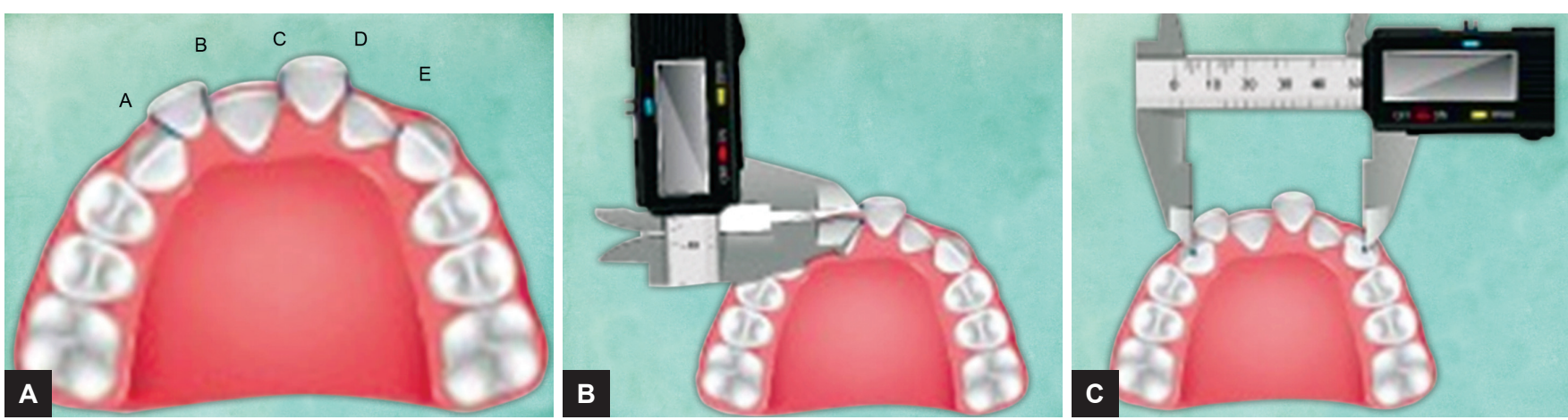

Figs $3 A$ to $C$ : Representative illustrations of the measurements in dental casts with the aid of a digital caliper: (A) Sum of the displacement $A+B+C+D+E$ following the Index Error; $(B)$ measurements with the caliper positioned parallel to the occlusal plane, evaluating only the tooth crowding of anatomical contact points; and $(\mathrm{C})$ the intercanine distance was measured with the caliper parallel to the occlusal plane and the reference was the canine cups tip

\section{Statistical Analysis}

Analyses were performed on GraphPad Prism Software (GraphPad Software, Inc., LaJolla, CA, USA). Measures were expressed as mean and the standard deviation of $\mathrm{T} 1$ and T2. The data were tested regarding the normal distribution by applying the Kolmogorov-Smirnov test. Because the distributions were normal, parametric tests could be used. Statistical significance was assessed using the paired t-test (systematic errors) for comparisons between $\mathrm{T} 1$ and $\mathrm{T} 2$, between the right and left sides and between the central incisor and lateral incisor. To investigate the correlations between variables, Pearson's correlation coefficient was used. A significance level of $5 \%$ $(p<0.05)$ was accepted for all tests. Variables in incisors and canines inclination as well as crowding and intercanine distance were remeasured at an average interval of 30 days. Ten subjects were randomly selected from 20 study participants to evaluate the casual and systematic errors. For evaluation of the random error the Dahlberg formula ${ }^{25}$ was used $\left(s=\sqrt{\Sigma \mathrm{d}^{2} / 2 \mathrm{n}}\right.$, where $\mathrm{d}=$ difference between the first and second measurements), and to calculate the systematic error, we used the paired t-test, where it was observed that the systematic error was insignificant in all the variables.

The average standard deviation of pre- (T1) vs posttreatment (T2) variation in the central and lateral incisors was $4.8^{\circ}$. Based on this value and adopting a $5 \%$ of significance level, power analysis showed that a sample size of at least 19 patients would give an $80 \%$ probability of detecting a minimum difference of $3.2^{\circ}$ of pre- vs posttreatment variation.

\section{RESULTS}

The results showed that the average score among the homologous teeth had no statistically significant differences in relation to the symmetry of the inclination movement on both sides (Tables 1 and 2). The protrusion
Table 1: Comparison between pre (T1) and posttreatment (T2) outcomes in all variables evaluated

\begin{tabular}{|c|c|c|c|c|c|c|}
\hline \multirow[b]{2}{*}{ Measurement } & \multicolumn{2}{|c|}{ (T1) } & \multicolumn{2}{|c|}{$(T 2)$} & \multirow[b]{2}{*}{ Diff. } & \multirow[b]{2}{*}{$p$-value } \\
\hline & Mean & $S D$ & Mean & $S D$ & & \\
\hline Upper arch & 7.38 & 3.94 & 0 & 0 & -7.38 & $<0.001^{*}$ \\
\hline Lower arch & 6.76 & 2.36 & 0 & 0 & -6.76 & $<0.001^{*}$ \\
\hline UID & 34.86 & 2.01 & 35.68 & 1.44 & 0.82 & $0.034^{*}$ \\
\hline LID & 25.61 & 1.53 & 27.57 & 1.24 & 1.96 & $<0.001^{*}$ \\
\hline URCI & 109.47 & 7.24 & 116.22 & 5.17 & 6.75 & $<0.001^{*}$ \\
\hline URLI & 112.02 & 6.42 & 116.70 & 4.21 & 4.68 & $<0.001^{*}$ \\
\hline URC & 107.38 & 7.75 & 106.88 & 5.37 & -0.50 & 0.646 \\
\hline ULCI & 109.89 & 6.78 & 116.24 & 5.56 & 6.35 & $<0.001^{*}$ \\
\hline ULLI & 112.30 & 6.69 & 117.43 & 5.38 & 5.13 & $<0.001^{*}$ \\
\hline ULC & 107.04 & 9.68 & 106.82 & 5.16 & -0.22 & 0.879 \\
\hline LLCI & 96.08 & 6.24 & 102.69 & 5.10 & 6.61 & $<0.001^{*}$ \\
\hline LLLI & 92.97 & 6.40 & 102.19 & 5.21 & 9.23 & $<0.001^{*}$ \\
\hline LLC & 95.25 & 3.79 & 99.15 & 5.57 & 3.90 & $0.013^{*}$ \\
\hline $\mathrm{LRCl}$ & 93.23 & 7.11 & 101.11 & 5.86 & 7.88 & $<0.001^{*}$ \\
\hline LRLI & 91.88 & 6.61 & 100.09 & 5.93 & 8.21 & $<0.001^{*}$ \\
\hline LRC & 92.78 & 6.09 & 96.63 & 5.35 & 3.86 & $0.004^{*}$ \\
\hline
\end{tabular}

*Statistically significant different $(p<0.05)$; UID: Upper intercanine distance; LID: Lower intercanine distance; URCI: Upper right central incisor; URLI: Upper right lateral incisor; URC: Upper right canine; ULCI: Upper left central incisor; ULLI: Upper left lateral incisor; ULC: Upper left canine; LLCI: Lower left central incisor; LLLI: Lower left lateral incisor; LLC: Lower left canine; LRCI: Lower right central incisor; LRLI: Lower right lateral incisor; LRC: Lower right canine

Table 2: Comparison between pre (T1) and posttreatment (T2) dental inclinations

\begin{tabular}{|c|c|c|c|c|c|c|}
\hline \multirow[b]{2}{*}{ Measurement } & \multicolumn{2}{|c|}{ (T1) } & \multicolumn{2}{|c|}{$(T 2)$} & \multirow[b]{2}{*}{ Diff. } & \multirow[b]{2}{*}{$p$-value } \\
\hline & Mean & $S D$ & Mean & $S D$ & & \\
\hline $\mathrm{UCl}$ & 109.68 & 6.56 & 116.23 & 5.27 & 6.55 & $<0.001^{*}$ \\
\hline ULI & 112.16 & 6.01 & 117.06 & 4.66 & 4.90 & $<0.001^{*}$ \\
\hline UC & 107.21 & 8.32 & 106.85 & 5.07 & -0.36 & 0.744 \\
\hline $\mathrm{LCl}$ & 94.66 & 6.00 & 101.90 & 5.26 & 7.24 & $<0.001^{*}$ \\
\hline LLI & 92.42 & 6.18 & 101.14 & 5.31 & 8.72 & $<0.001^{*}$ \\
\hline LC & 94.01 & 3.91 & 97.89 & 4.83 & 3.88 & $<0.001^{*}$ \\
\hline
\end{tabular}

*Statistically significant different $(p<0.05)$; UCl: Upper central incisor; ULI: Upper lateral incisor; UC: Upper canine; LCl: Lower central incisors; LLI: Lower lateral incisor; LC: Lower canine 
Table 3: Correlation between dental crowding and intercanine distance in the pretreatment (T1) related to the variations in the dental inclinations during treatment

\begin{tabular}{lcl}
\hline Correlation & $r$ & $p$-value \\
\hline Upper arch $\times$ UCl dental inclination & 0.01 & 0.956 \\
Upper arch $\times$ ULI dental inclination & 0.43 & 0.057 \\
Upper arch $\times$ UC dental inclination & -0.59 & $0.006^{*}$ \\
Lower arch $\times$ LCl dental inclination & 0.39 & 0.093 \\
Lower $\operatorname{arch} \times$ LLI dental inclination & 0.25 & 0.287 \\
Lower arch $\times$ LC dental inclination & -0.30 & 0.195 \\
UICD $\times$ UCl dental inclination & 0 & 0.998 \\
UICD $\times$ ULI dental inclination & 0.04 & 0.873 \\
UICD $\times$ UC dental inclination & -0.55 & $0.015^{*}$ \\
LICD $\times$ LCl dental inclination & -0.13 & 0.585 \\
LICD $\times$ LLI dental inclination & -0.28 & 0.237 \\
LICD $\times$ LC dental inclination & -0.44 & 0.062 \\
\hline COre
\end{tabular}

${ }^{*}$ Correlation statistically significant different $(p<0.05)$; UCl: Upper central incisor; ULI: Upper lateral incisor; UC: Upper canine; LCI: Lower central incisors; LLI: Lower lateral incisor; LC: Lower canine; UICD: Upper intercanine distance; LICD: Lower intercanine distance

was the most expressive movement, where the inclination average was: $6.55^{\circ}$ for the upper central incisors and $7.24^{\circ}$ to the lower incisors. The upper $\left(4.90^{\circ}\right)$ and lower $\left(8.72^{\circ}\right)$ lateral incisors also obtained positive inclinations.

The lower canines maintained positive inclination as the lower incisors with $3.88^{\circ}$ of average (Table 2), and an increase in intercanine width of $1.96 \mathrm{~mm}$ were observed (Table 1). In contrast, the upper canines showed an opposite movement to the upper incisors, assuming a negative inclination with $-0.36^{\circ}$ of average (Table 2 ) with an increase of $0.82 \mathrm{~mm}$ in the intercanine distance (Table 1 ), assuming negative correlation between crowding and the initial intercanine distance (Table 3).

\section{DISCUSSION}

Unlike classic examination in orthodontics ${ }^{19}$ where the $3 \mathrm{D}$ teeth evaluations are difficult to achieve, the establishment of CBCT image acquisition allowed precisely and accurate methods of analysis in specific areas, particularly related to the incisors and canines inclinations, which was not possible with the aid of lateral radiograph of the face. ${ }^{2,20,26}$ Indeed, anterior teeth position changes obtained by passive self-ligating brackets evaluated with $\mathrm{CBCT}$ scans are scarce in the scientific literature, and few studies have assessed the mandibular changes with self-ligating brackets and $\mathrm{CBCT} .{ }^{16}$

The CBCT has lower radiation doses than conventional computed tomography (CT). ACT scan of the head presents an effective radiation dose of about 7 to 12 times higher than the i-Cat CBCT scanner, considering similar FOV. ${ }^{27-29}$ Therefore, CBCT is the only 3D method with lower exposure, able to provide information with qualified accuracy on the changes in alveolar bone support resulting from the orthodontic tooth movement in vivo. ${ }^{30}$ Nevertheless, a low-dose CBCT-scanner was used. The patients in the present study received at least 10 times less radiation than the dose for an equivalent examination performed with medical CT scanner. ${ }^{29}$ The use of adequate protection (protective thyroid collar) resulted in reduced radiation levels to the patient, ${ }^{31}$ and such radiation protection methods were in the accomplishment of this work.

Here, the Little irregularity index ${ }^{23}$ was chosen to measure the teeth crowding in the dental casts, as an easily reproducible and reliable method as well as the method adopted by Shapiro, ${ }^{24}$ who used the cusp tip as reference points to measure changes in the intercanine distance. These two methods of analysis are quantitative methods in assessing anterior teeth irregularities and to evaluate pretreatment and posttreatment changes.

High flexibility wires, such as nickel-titanium were first introduced in orthodontics in the 1980s, allowing that poorly positioned teeth even with space deficiency could be included in the mechanics since the beginning of leveling, ${ }^{3,32}$ generating a greater movement toward the buccal direction. ${ }^{1-4,18}$

In contrast, previous study ${ }^{5}$ in the literature supports the slow expansion without excessive protrusion of the incisors. The work of $\mathrm{Chen}^{\mathrm{at}} \mathrm{al}^{7}$ showed a reduction of $1.5^{\circ}$ in the incisors inclination for cases treated with passive self-ligating brackets. These results parallel observations made by Damon's, ${ }^{17}$ reaffirming that as thermoactivated alloys propagate light forces, and the time is directly related to the strength, the inclination movement tends to be reduced.

In contrast, Vajaria et $\mathrm{al}^{4}$ demonstrated after treatment with passive self-ligating system that the lower incisors exhibited significant inclination (advanced and proclined) toward the buccal direction, even with the use of low-width wires. These results indicate that the incisors inclination after treatment did not differ significantly when Damon brackets and conventional brackets were compared. ${ }^{4}$ Accordingly, Pandis et al ${ }^{3}$ concluded that selfligating brackets appear to be equally efficient in delivering torque to the upper incisors similar to conventional brackets in extraction and nonextraction sites. Based on the results obtained in this study, the lip bumper theory, ${ }^{33}$ in which the reduction of the incisors buccal movement results in freedom of wires inside the passive brackets channel when associated with muscular action, could raises some doubts about this concept.

When dental crowding are present in the maxillary and/or mandibular arches, even in moderate crowding cases, the treatment without extraction or expansion causes buccal teeth inclinations. ${ }^{1,4,18}$ The MBT prescription, used in the present study presents positive values for central $\left(+17^{\circ}\right)$ and lateral $\left(+10^{\circ}\right)$ incisors torque. At the 
end of leveling, 10 weeks after the installation of $0.019^{\prime \prime}$ $\times 0.025^{\prime \prime}$ steel wire, patients had an inclination mean increase in the central and lateral incisors of $6.55^{\circ}$ and $4.90^{\circ}$ respectively. In the lower arch, even with the MBT prescription presenting negative values for the incisors $\left(-6^{\circ}\right)$, the central incisors had an average increase of $7.24^{\circ}$, while the lateral incisors increased $8.7^{\circ}$. Importantly, there was a tendency to a contrary effect for the upper canines, where they remained in the same position or had small increase in their palatal inclinations, while the lower canines increased buccal inclination (Tables 1 and 2).

Comparing the amount of initial crowding over the final incisors inclination, the present study did not indicate correlation between the initial crowding degree and final amount of incisors inclination, which are in contrast to the findings of Fleming et $\mathrm{al}^{18}$ that observed a positive correlation between the amount of initial crowding and the final inclination of the lower incisors in subjects treated without extractions and with passive self-ligating brackets (Smartclip). These findings could potentially be explained to the differences in the methodology employed, in which Fleming et $\mathrm{al}^{18}$ included 11 contact points in the mandibular arch and considered all three dimensions: Mesiodistal, buccolingual, and vertical whereas our study only measured the distance with a digital caliper placed parallel to the occlusal plane.

In a previous systematic review, Burke et $\mathrm{al}^{34}$ aimed to assess the longitudinal stability of mandibular intercanine distance. Linear and dimensional changes in intercanine transverse distance were compared before treatment (T1), immediately after treatment (T2), and after the end of treatment (T3). The results showed that, on average, intercanine distance increases from 1 to $2 \mathrm{~mm}$, regardless of the treatment and the initial molar classification. Cattaneo et $\mathrm{al}^{2}$ evaluated the changes in the mandibular incisors and canine's inclination between passive self-ligating brackets (Damon) and active self-ligating brackets (In-Ovation). They found that the average increase of the canine's inclination was $3.9^{\circ}$ and $3.2^{\circ}$ for passive and active brackets respectively. With regard to the intercanine width, 2.0 and $1.6 \mathrm{~mm}$ increase were observed for the active and passive brackets respectively. Accordingly, our findings close resemble observations made by Cattaneo et $\mathrm{al}^{2}{ }^{2}$ in which the lower canines maintained positive inclination with $3.88^{\circ}$ of average (Table 2), and an increase in intercanine width of $1.96 \mathrm{~mm}$ using passive self-ligating brackets.

In a recent in vitro study, Maltagliati et $\mathrm{al}^{5}$ analyzed transversal changes in dental arches from nonextraction treatment with self-ligating brackets in 58 pairs of dental casts. The authors showed that intercanine distance had a mean increase of $0.75 \mathrm{~mm}$ in the upper arch and 1.96 $\mathrm{mm}$ in the mandibular teeth. Here, we obtained similar results in regard to the increased intercanine width, i.e.,
0.82 and $1.96 \mathrm{~mm}$ for the upper and lower archer respectively, keeping correlation with increased intercanine distance. The inclination movement of the lower canines showed an average increase of $3.88^{\circ}$ even using a prescription presenting $0^{\circ}$ of inclination. The upper canines presented with distinctive movements, where increase in the intercanine distance and decrease in the average inclination $\left(-0.36^{\circ}\right)$ was observed. Such movement would be justified considering the canine as a "support tooth", exerting sustenance function for the inclination movement of the incisors.

When alignment and leveling movement are performed, without the use of diagrammed wires, stripping, or extractions, the canines play an important role as support tooth, which tends to stay as close as to its original position. The incisors are prone to buccal direction, which means, different primary movements especially for the upper arch. This could explain the fact that intercanine distance in the upper arch did not increase and the inclination decreased.

\section{CONCLUSION}

Orthodontic treatment protocol with self-ligating brackets in patients with dental crowding treated without extraction and/or stripping produced increments on the buccal inclination of the upper and lower incisors in similar amounts and symmetrically, without correlation with the amount of crowding present at the start of treatment.

The canine inclination was different for the upper $\left(-0.36^{\circ}\right)$ and lower $\left(3.88^{\circ}\right)$ arches. The intercanine distance increased very little $(0.82$ and $1.96 \mathrm{~mm}$ for upper and lower arches respectively), showing a negative correlation between inclination of canines and intercanine distance to the upper arch.

\section{CLINICAL SIGNIFICANCE}

When taking into account the self-ligating brackets, the amount of initial dental crowding is not a limitation factor that could increase the buccal inclination of the anterior teeth.

\section{ACKNOWLEDGMENT}

Fapesp process number 2012/21393-8.

\section{REFERENCES}

1. Atik E, Ciger S. An assessment of conventional and selfligating brackets in class I maxillary constriction patients. Angle Orthod 2014 Jul;84(4):615-622.

2. Cattaneo PM, Salih RA, Melsen B. Labiolingual root control of lower anterior teeth and canines obtained by active and passive self-ligating brackets. Angle Orthod 2013 Jul;83(4): 691-697. 
3. Pandis N, Strigou S, Eliades T. Maxillary incisor torque with conventional and self-ligating brackets: a prospective clinical trial. Orthod Craniofac Res 2006 Nov;9(4):193-198.

4. Vajaria R, BeGole E, Kusnoto B, Galang MT, Obrez A. Evaluation of incisor position and dental transverse dimensional changes using the Damon system. Angle Orthod 2011 Jul;81(4):647-652.

5. Maltagliati LA, Myiahira YI, Fattori L, Filho LC, Cardoso M. Transversal changes in dental arches from non-extraction treatment with self ligating brackets. Dental Press J Orthod 2013 May-Jun;18(3):39-45.

6. Basciftci FA, Akin M, Ileri Z, Bayram S. Long-term stability of dentoalveolar, skeletal, and soft tissue changes after non-extraction treatment with a selfligating system. Korean J Orthod 2014 May;44(3):119-127.

7. Chen SS, Greenlee GM, Kim JE, Smith CL, Huang GJ. Systematic review of self-ligating brackets. Am J Orthod Dentofacial Orthop 2010 Jun;137(6):726.e1-726.e18; discussion 726-727.

8. Leite V, Conti AC, Navarro R, Almeida M, Oltramari-Navarro P, Almeida R. Comparison of root resorption between selfligating and conventional preadjusted brackets using cone beam computed tomography. Angle Orthod 2012 Nov;82(6):1078-1082.

9. Othman SA, Mansor N, Saub R. Randomized controlled clinical trial of oral health-related quality of life in patients wearing conventional and self-ligating brackets. Korean J Orthod 2014 Jul;44(4):168-176.

10. Khambay B, Millett D, McHugh S. Evaluation of methods of archwire ligation on frictional resistance. Eur J Orthod 2004 Jun;26(3):327-332.

11. Cordasco G, Lo Giudice A, Militi A, Nucera R, Triolo G, Matarese $\mathrm{G}$. In vitro evaluation of resistance to sliding in self-ligating and conventional bracket systems during dental alignment. Korean J Orthod 2012 Aug;42(4):218-224.

12. Lee SM, Hwang CJ. A comparative study of frictional force in self-ligating brackets according to the bracket-archwire angulation, bracket material, and wire type. Korean J Orthod 2015 Jan;45(1):13-19.

13. Fleming PS, Johal A. Self-ligating brackets in orthodontics. A systematic review. Angle Orthod 2010 May;80(3):575-584.

14. Pandis N, Polychronopoulou A, Eliades T. Self-ligating vs conventional brackets in the treatment of mandibular crowding: a prospective clinical trial of treatment duration and dental effects. Am J Orthod Dentofacial Orthop 2007 Aug;132(2):208-215.

15. Fleming PS, DiBiase AT, Sarri G, Lee RT. Comparison of mandibular arch changes during alignment and leveling with 2 preadjusted edgewise appliances. Am J Orthod Dentofacial Orthop 2009 Sep;136(3):340-347.

16. Almeida MR, Futagami C, Conti AC, Oltramari-Navarro PV, Navarro Rde L. Dentoalveolar mandibular changes with selfligating versus conventional bracket systems: a CBCT and dental cast study. Dental Press J Orthod 2015 May-Jun;20(3): 50-57.

17. Damon DH. The rationale, evolution and clinical application of the self-ligating bracket. Clin Orthod Res 1998 Aug;1(1):52-61.
18. Fleming PS, DiBiase AT, Sarri G, Lee RT. Efficiency of mandibular arch alignment with 2 preadjusted edgewise appliances. Am J Orthod Dentofacial Orthop 2009 May;135(5):597-602.

19. Gracco A, Luca L, Bongiorno MC, Siciliani G. Computed tomography evaluation of mandibular incisor bony support in untreated patients. Am J Orthod Dentofacial Orthop 2010 Aug;138(2):179-187.

20. Peck JL, Sameshima GT, Miller A, Worth P, Hatcher DC. Mesiodistal root angulation using panoramic and cone beam CT. Angle Orthod 2007 Mar;77(2):206-213.

21. Baumrind S, Miller D, Molthen R. The reliability of head film measurements. 3. Tracing superimposition. Am J Orthod 1976 Dec;70(6):617-644.

22. Guedes FP, Capelozza Filho L, Garib DG, Nary Filho H, Borgo EJ, Cardoso Mde A. Impact of orthodontic decompensation on bone insertion. Case Rep Dent 2014;2014:341752.

23. Little RM. The irregularity index: a quantitative score of mandibular anterior alignment. Am J Orthod 1975 Nov;68(5): 554-563.

24. Shapiro PA. Mandibular dental arch form and dimension. Treatment and postretention changes. Am J Orthod 1974 Jul;66(1):58-70.

25. Houston WJ. The analysis of errors in orthodontic measurements. Am J Orthod 1983 May;83(5):382-390.

26. Scarfe WC, Farman AG, Sukovic P. Clinical applications of cone beam computed tomography in dental practice. J Can Dent Assoc 2006 Feb;72(1):75-80.

27. Grunheid T, Kolbeck Schieck JR, Pliska BT, Ahmad M, Larson BE. Dosimetry of a cone beam computed tomography machine compared with a digital X-ray machine in orthodontic imaging. Am J Orthod Dentofacial Orthop 2012 Apr;141(4):436-443.

28. Ludlow JB, Davies-Ludlow LE, Brooks SL, Howerton WB. Dosimetry of 3 CBCT devices for oral and maxillofacial radiology: CB Mercuray, NewTom 3G and i-CAT. Dentomaxillofac Radiol 2006 Jul;35(4):219-226.

29. Ludlow JB, Ivanovic M. Comparative dosimetry of dental CBCT devices and 64-slice CT for oral and maxillofacial radiology. Oral Surg Oral Med Oral Pathol Oral Radiol Endod 2008 Jul;106(1):106-114.

30. Kapila S, Conley RS, Harrell WE Jr. The current status of cone beam computed tomography imaging in orthodontics. Dentomaxillofac Radiol 2011 Jan;40(1):24-34.

31. Qu X, Li G, Zhang Z, Ma X. Thyroid shields for radiation dose reduction during cone beam computed tomography scanning for different oral and maxillofacial regions. Eur J Radiol 2012 Mar;81(3):e376-e380.

32. Pandis N, Polychronopoulou A, Eliades T. Active or passive self-ligating brackets? A randomized controlled trial of comparative efficiency in resolving maxillary anterior crowding in adolescents. Am J Orthod Dentofacial Orthop 2010 Jan;137(1):12.e1-12.e6; discussion 12-13.

33. Damon DH. The Damon low-friction bracket: a biologically compatible straightwire system. J Clin Orthod 1998 Nov;32(11):670-680.

34. Burke SP, Silveira AM, Goldsmith LJ, Yancey JM, Van Stewart A, Scarfe WC. A meta-analysis of mandibular intercanine width in treatment and postretention. Angle Orthod 1998 Feb;68(1):53-60. 